\title{
Alper Syndrome
}

National Cancer Institute

\section{Source}

National Cancer Institute. Alper Syndrome. NCI Thesaurus. Code C35257.

A rare genetic syndrome with an autosomal recessive pattern of inheritance. It is caused by a mutation in the gene for the mitochondrial DNA polymerase POLG. Clinical signs are usually not present at birth but develop within the first two years of life and include hypoglycemia from underlying liver dysfunction, failure to thrive, spasticity, myoclonus and seizures. The clinical course follows a progression of neurologic disability and hepatic failure. The prognosis is poor with survival outside the first decade unlikely. 